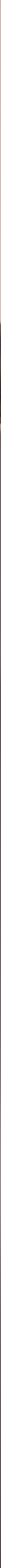




\section{Pulmonary rehabilitation: a year in review}

\section{Educational aims}

I To present innovative papers from the field of pulmonary rehabilitation published in 2005 and to discuss the important points and conclusions from each.

\section{Summary}

This paper summarises the findings of important papers that have been published over the last year.

Two recently published studies have addressed the effects of different durations of pulmonary rehabilitation programme (PRP), indicating the greater benefits obtained by longer programmes.

Three other papers have described the effects of pulmonary rehabilitation on pulmonary mechanics and oxidative stress, indicating that exercise is able to reduce the mechanical disadvantage of the respiratory system and some aspects of oxidative stress associated with chronic obstructive pulmonary disease (COPD).

Other papers have discussed the effects of a number of breathing strategies, such as pursed-lip breathing (PLB), or training techniques, such as arm or inspiratory muscle training.

Finally, the addition of drug therapy to pulmonary rehabilitation and the role of outcome measures in providing rehabilitation programmes were examined in two other studies.

COPD is a leading cause of death, and a major medical and increasing economic problem [1]. Pulmonary rehabilitation has been established as a means of enhancing standard therapy to alleviate symptoms and optimise function, independent of the disease stage. Randomised controlled studies and meta-analyses have shown that intensive, multidisciplinary PRPs are an effective intervention in the short and long term, comprising improved exercise tolerance, symptoms and health-related quality of life (HRQL), and a decrease in healthcare expenditures [2]. The benefits appear to decline after 6-12 months following the formal programme [3]. Benefits of HRQL appear better preserved than exercise performance, and may still be identified up to 2 years after the intervention [4]. This is a review of the most significant original papers published in 2005 on pulmonary rehabilitation. Research was conducted using PubMed between January 1 to November 31, 2005, with the search criterion "pulmonary rehabilitation". A total of 187 articles were extracted, 36 of which were reviews or editorials.
N. Ambrosino

M. Serradori

Pulmonary Unit, Cardio-Thoracic Dept, University Hospital, Pisa, Italy.

\section{Correspondence:}

N. Ambrosino

U.O. Pneumologia-Dipartimento Cardio-Toracico

Azienda Ospedaliero-Universitaria Pisana

Via Paradisa 2

Cisanello

56124 Pisa

Italy

Fax: 39050996779

E-mail:n.ambrosino@ao-

pisa.toscana.it 


\section{Setting and organisation of PRPs}

The optimal length of a PRP is of interest. Pulmonary rehabilitation can be delivered in a variety of structured programmes that may have an influence on the degree or duration of longterm benefit. Short-term in-patient programmes may result in physical performance improvement within 2 weeks [5]. Strict comparisons of dose response or duration of benefits from different PRPs have not yet been made, and the rate of improvement in exercise performance and HRQL may differ. It is possible that improvements may continue to develop after the completion of a programme, but observation of these effects has not yet been determined.

Two recently published studies in this field add little to this issue. In an observational prospective cohort trial, Rossi et al. [6] assessed the clinical effectiveness of a PRP including exercise training after 10 (T10) or 20 (T20) consecutive sessions in 25 COPD outpatients (mean forced expiratory volume in one second (FEV1) $64 \%$ predicted). The maximal achieved load on a cycle ergometer, the maximal and isoload dyspnoea and leg fatigue on a Borg scale, the 6-minute walk distance (6MWD) and the HRQL (as assessed using the St George's Respiratory Questionnaire (SGRQ)) were recorded at baseline, T10 and T20. There was a mean significant difference between changes at T20 and T10 for 6MWD, and the total, activity and symptoms SGRQ scores. The percentage of patients who improved was greater at T20 compared with T10 for peak workload, 6MWD and total SGRQ. These authors concluded that a 10-session course of PRP provides only limited clinically significant changes of outcome measures when compared with a 20-session course in outpatients with COPD of mild-to-moderate severity.

In a multicentre study, VerRILL et al. [7] evaluated 309 females and 281 males with chronic lung disease. All 6MWD tests and health surveys were administered prior to and immediately following 12 and 24 weeks of supervised PRP participation. Scores from the 6MWD tests, the Ferrans and Powers quality of life indexpulmonary version III (QLI), the Medical Outcomes Study 36-item short form (SF-36), and the University of California at San Diego shortness of breath questionnaire (SOBQ) were compared at PRP entry, and at 12 and 24 weeks. The study entry and follow-up SF-36 physical and mental component summary scores, the QLI health/function and overall scores, and the SOBQ scores were also compared with the 6MWD test scores. The mean summary scores on the SF-36 and the QLI increased after 12 weeks of PRP, and improvements were maintained by 24 weeks of PRP participation. Scores on the SOBQ improved after 12 weeks among the shortterm participants, but not until after 24 weeks among the long-term participants. The 6MWD test performance improved after 12 weeks and again from 12-24 weeks in the long-term participants. No relevant relationships were found between 6MWD scores and the summary scores of the administered surveys.

\section{Physiological and biological effects of pulmonary rehabilitation}

In COPD, ventilation during exercise is often higher than expected because of increased dead space ventilation, impaired gas exchange and increased ventilatory demands relating to deconditioning and peripheral muscle dysfunction. Furthermore, maximal ventilation during exercise is often limited by the mechanical constraints imposed by the lung pathophysiology. Prominent among these constraints and typically seen in emphysematous patients is the delay of normal emptying of the lungs during expiration due to flow limitation, which is aggravated during exercise. This leads to dynamic hyperinflation [8], resulting in increased work of breathing, increased load on the respiratory muscles [9] and the intensified perception of respiratory discomfort.

PLANKeEl et al. [10] retrospectively analysed the change in exercise capacity after PRP in nonhypoxaemic COPD patients. Patients were classified into the following subgroups based on the primary limitation seen on initial exercise testing: ventilatory limited; cardiovascular limited; mixed ventilatory/cardiovascular limited; and non-cardiopulmonary limited. Outcomes among subgroups were then compared. In the whole study population, PRP led to increased timed walk distance and maximal oxygen consumption. Age, ventilatory reserve at peak exercise and exercise arterial oxygen pressure were selected as individual predictors of improvement in maximal oxygen consumption. Maximal oxygen consumption increased in all four subgroups. The improvement in maximal oxygen consumption 
was greater in the cardiovascular-limited subgroup than in the ventilatory-limited subgroup. Timed walk distance improved to a similar degree in all subgroups. PLANKEEL et al. [10] concluded that patients with non-ventilatory exercise limitations experience the greatest increase in maximal oxygen consumption after PRP. However, even patients with severe ventilatory limitation can improve exercise tolerance with PRP.

Ponszasz et al. [11] studied COPD patients (mean FEV1 36\% pred) who undertook a highintensity cycle ergometer exercise training programme for 45 minutes, three times a week for 7 weeks. Before and after training, the patients performed an incremental exercise test to maximum and a constant work rate test on a cycle ergometer at $75 \%$ of the peak work rate obtained in the pre-training incremental test. Ventilatory variables were measured breath by breath, and inspiratory capacity (IC) was measured every 2 minutes to assess changes in endexpiratory lung volume. After training, the increase in peak oxygen uptake was not statistically significant; however, the peak work rate increased. For the constant work rate test, performed at the same work rate both before and after training, ventilation and breathing frequency were lower after training and IC was greater, signifying decreased hyperinflation. The increase in IC at the point of termination in the shortest constant work rate test for each individual (defined as isotime) correlated well with both the decreased breathing frequency and with the increase in constant work rate exercise endurance. The authors concluded that exercise training in patients with severe COPD dramatically improves submaximal exercise endurance. Decreased dynamic hyperinflation may, in part, mediate the improvement in exercise endurance by delaying the attainment of a critically high inspiratory lung volume.

Several studies have shown an increased systemic oxidative stress response after strenuous exercise in COPD patients [12]. In COPD patients, MERCKEN et al. [13] studied the effect of exercise at different intensities, as well as the effect of intensive supervised pulmonary rehabilitation on oxidative stress. COPD patients and healthy age-matched control subjects performed a maximal and submaximal exercise cycle ergometry test at $60 \%$ of peak workload. Patients with COPD performed these tests before and after 8 weeks of PRP. Measurements were taken before, immediately after and 4 hours

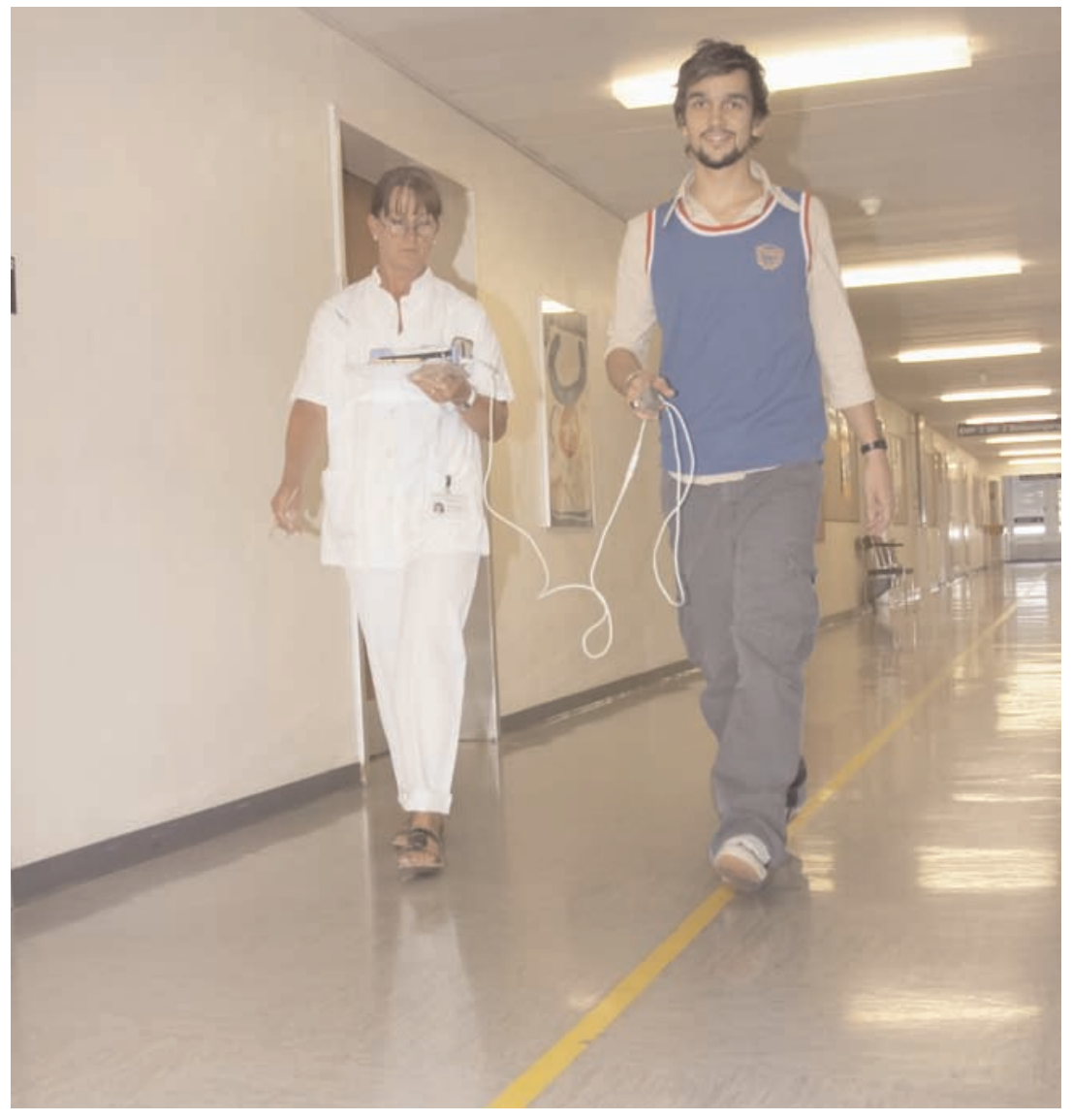

after both exercise tests. At rest, increased oxidative stress was observed in patients compared with control subjects, as measured by urinary malondialdehyde (MDA) and hydrogen peroxide $\left(\mathrm{H}_{2} \mathrm{O}_{2}\right)$ in breath condensate. In healthy control subjects, a significant increase in urinary MDA was observed 4 hours after both exercise tests, whereas $\mathrm{H}_{2} \mathrm{O}_{2}$ significantly increased immediately after maximal exercise. In COPD patients before rehabilitation, reactive oxygen speciesinduced DNA damage in peripheral blood mononuclear cells, urinary MDA and plasma uric acid were significantly increased after both exercise tests, whereas no significant increase was observed in plasma MDA. In contrast, exhaled $\mathrm{H}_{2} \mathrm{O}_{2}$ was only significantly increased after maximal exercise. Although after rehabilitation peak workload was increased by $24 \%$, a similar oxidative stress response was found. Remarkably, a decrease in reactive oxygen species-induced DNA damage was detected after exercise at submaximal intensity, despite increased exercise duration of $73 \%$. In summary, patients with COPD had increased pulmonary and systemic oxidative stress both at rest and induced by exercise. In addition, pulmonary rehabilitation increased exercise capacity and was associated 
with reduced exercise-induced oxidative stress. The study by MERCKEN et al. [13] is an important paper, indicating the possible biological effects of PRP. Other reported biological effects of PRP include an increase in the level of exhaled nitric oxide [14]. Therefore, there is growing evidence that the physiological and clinical effects of pulmonary rehabilitation may be mediated by effects on biological conditions associated with COPD.

\section{Drug therapy and pulmonary rehabilitation}

Another important issue regarding pulmonary rehabilitation is the possible role of drug therapy in enhancing the effects. Tiotropium is a oncedaily, inhaled anticholinergic bronchodilator that provides sustained 24-hour improvements in airflow and lung hyperinflation reduction. In a randomised, double-blind, placebo-controlled trial [15], tiotropium was administered to COPD patients (mean FEV1 34\% pred) participating in 8 weeks of PRP (treadmill training three times a week; $\geq 30$ min per session). The study drug was administered 5 weeks prior to, 8 weeks during, and 12 weeks following PRP. The primary endpoint was treadmill walking endurance time at $80 \%$ of maximum speed attained in an initial incremental test. The transition dyspnoea index (TDI), SGRQ and rescue salbutamol use were secondary end-points. Mean endurance time prior to, at the end of and 12 weeks after PRP progressively increased. Mean TDI focal scores at the end of PRP and at 12 weeks after PRP were greater for tiotropium than for placebo. Relative to placebo, tiotropium improved SGRQ total scores at the end of PRP and, to a greater extent, at 12 weeks after PRP. Mean salbutamol use declined following PRP plus tiotropium, compared with PRP alone.

\section{Breathing strategies}

Breathing strategies refer to a range of techniques, including $P L B$, active expiration, diaphragmatic breathing, adapting specific body positions and coordinating paced breathing with activities. These techniques aim to improve regional ventilation, gas exchange, respiratory muscle function, dyspnoea, exercise tolerance and HRQL. PLB attempts to prolong active expiration through half-opened lips, thus helping to prevent airway collapse. Compared to spontaneous breathing, PLB reduces respiratory rate, dyspnoea and arterial carbon dioxide tension, while improving tidal volume and oxygen saturation in resting conditions [16]. Although these have not been convincingly demonstrated to result in enhanced exercise performance, many patients with chronic lung disease use this technique instinctively and have reported decreased dyspnoea with its use.

SPAHIJA et al. [17] investigated the effect of PLB on breathing pattern, respiratory mechanics, operational lung volumes and dyspnoea in patients with COPD (mean FEV1 50\% pred). PLB promoted a slower and deeper breathing pattern both at rest and during exercise. Whereas patients had no dyspnoea with or without PLB at rest, dyspnoea was variably affected by PLB across patients during exercise. Changes in the individual dyspnoea scores with PLB during exercise were significantly correlated with changes in the end-expiratory lung volume values estimated from IC manoeuvres (as a percentage of total lung capacity) and with changes in the mean inspiratory ratio of pleural pressure to the maximal static inspiratory pressure-generating capacity $\left(P_{\text {capl }}\right)$, measured using an oesophageal balloon, where $P$ capl was determined over the range of inspiratory lung volumes and adjusted for flow. SPAHIJA et al. [17] concluded that PLB can have a variable effect on dyspnoea when performed volitionally during exercise by COPD patients. The effect of PLB on dyspnoea is related to the combined change that it promotes in the tidal volume and end-expiratory lung volume, and their impact on the available capacity of the respiratory muscles to meet the demands placed on them in terms of pressure generation. As with the development of all aspects of the patient's self-management, breathing strategies must be individualised. Patients will usually adopt the techniques that are most effective in reducing symptoms.

\section{Respiratory muscle training}

In some studies, the addition of inspiratory muscle training to standard exercise training in patients with poor initial inspiratory muscle strength has been shown to improve exercise capacity more than exercise training alone. In patients with less respiratory muscle weakness, evidence for the addition of inspiratory muscle training to regular exercise training is lacking. 
Three types of inspiratory muscle training have been reported: inspiratory resistive training, threshold loading and normocapnic hyperpnoea. At present, there are no data to support one method over the other. Hyperpnoea training has been shown to have beneficial effects in COPD patients [18].

MADOR et al. [19] have determined whether hyperpnoea training, when added to an endurance exercise training programme, would lead to additional benefits compared with endurance training alone in COPD patients entering an 8-week outpatient PRP. Patients (mean FEV $145 \%$ pred) were randomised to combined therapy or endurance training. Peak exercise capacity, exercise endurance time during constant workload cycle exercise, 6MWD, $\mathrm{HRQL}$ as measured by the chronic respiratory questionnaire, respiratory muscle strength and endurance, and quadriceps fatigability were measured before and after endurance or combined training. After rehabilitation, peak exercise capacity, exercise endurance time, 6MWD and $\mathrm{HRQL}$ all increased in both groups, but there was no significant difference in the extent of improvement between groups. Mean respiratory muscle endurance increased to a significantly greater extent in the combined therapy group. Respiratory muscle strength was significantly increased, and quadriceps fatigability was significantly reduced after rehabilitation in the combined therapy group although not in the endurance training group, but the difference between groups did not reach statistical significance. The authors concluded that the endurance of the respiratory muscles can be improved by specific training beyond that achieved by endurance training alone in COPD patients. However, this improvement did not translate into additional improvement in quality of life or exercise performance.

BECKERMAN et al. [20] assessed the long-term benefits of inspiratory muscle training on inspiratory muscle strength, exercise capacity, perception of dyspnoea, $\mathrm{HRQL}$, primary-care use and hospitalisations in COPD patients with FEV1 $<50 \%$ pred who were randomised into a group that received inspiratory muscle training for 1 year, and a control group who received training with a very low load. There was a statistically significant increase in inspiratory muscle strength and 6MWD, a decrease in the mean Borg score during breathing against resistance, and improvement in the HRQL scores in the training group but not in the control group. At the end of the training year, these changes were maintained; in addition, a decrease in primary healthcare use and hospitalisation days was observed.

\section{Arm training}

Most patients with stable moderate COPD complain of dyspnoea during activities involving the upper extremities. In these patients, thoracoabdominal dyssynchrony and an increase in oxygen consumption and minute ventilation $(V E)$

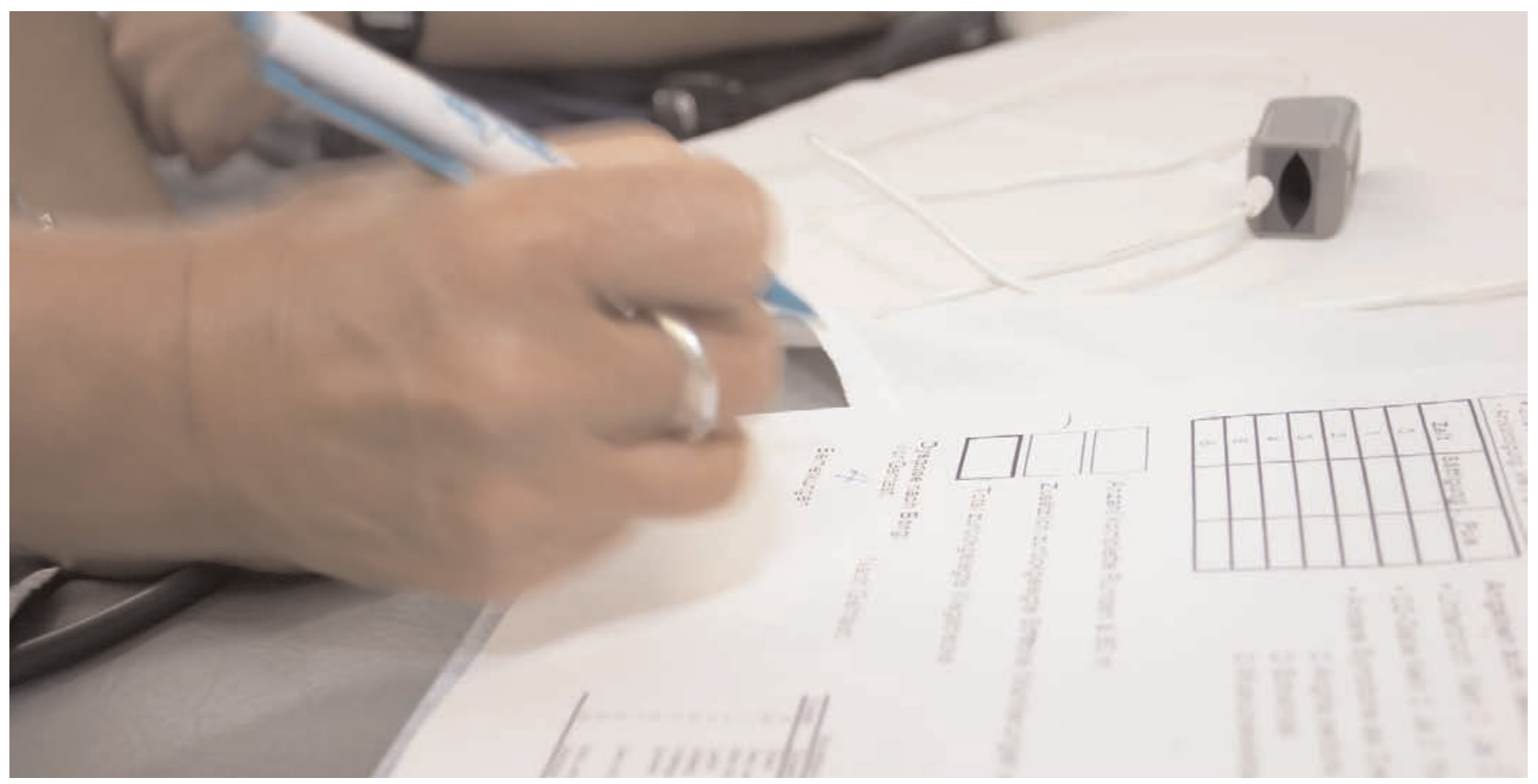




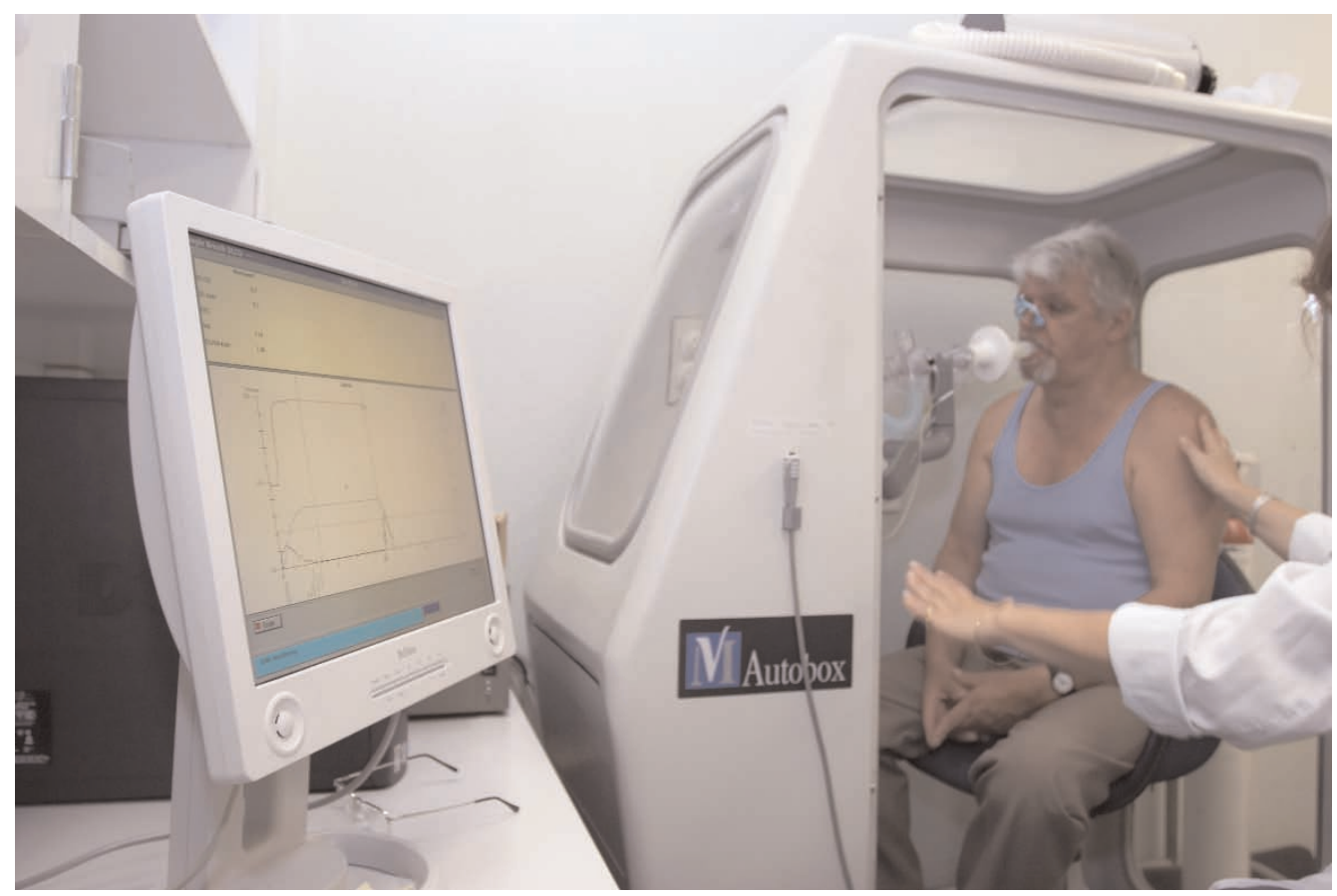

are observed during unsupported arm activity in comparison with leg middle-intensity exercise. One explanation for this is that, with increasing hyperinflation, inspiratory muscle predominance may shift from the diaphragm to the accessory inspiratory muscles, including some muscles of the shoulder girdle that contribute to both upperextremity positioning and pulmonary ventilation. In line with this concept, upperextremity training would yield greater improvement (although not significantly) in maximal inspiratory pressure. Few controlled studies have evaluated the effects of upper-limb training [21].

GIGLIOTTI et al. [22] have ascertained the following in patients with COPD: 1) whether and to what extent upper-limb exercise results in dynamic hyperinflation; and 2) the mechanism whereby an arm training programme reduces arm effort and dyspnoea. Twelve patients with moderate-to-severe COPD were tested during incremental, symptom-limited arm exercise after a non-interventional control period (before the arm training programme) and after the arm training programme. Exercise testing was performed using an arm ergometer. Oxygen uptake, carbon dioxide output, $V_{E}$, tidal volume and respiratory rate were measured continuously during the tests. IC, exercise dyspnoea and arm effort using a Borg scale were assessed at each step of exercise. Arm exercise resulted in a significant decrease in IC, and significant positive relationships of IC with an increase in oxygen uptake and exercise dyspnoea and arm effort.
The results of the arm training programme were as follows: 1) a significant increase in exercise capacity; 2) no change in the relationships of exercise dyspnoea and arm effort with $V_{E}$ and IC, and of IC with oxygen uptake; 3) at a standardised work rate, $V E$, exercise dyspnoea and arm effort significantly decreased, whereas the decrease in IC was significantly less than before the arm training programme (the decrease in $V \mathrm{E}$ was accomplished primarily by a decrease in respiratory rate); and 4) at standardised $V E$, exercise dyspnoea and arm effort decreased significantly. Arm exercise resulted in the association of dynamic hyperinflation, exercise dyspnoea and arm effort in COPD patients. Thus, an arm training programme increases arm endurance, modulates dynamic hyperinflation and reduces symptoms.

In a multicentre prospective, randomised, controlled study, PORTA et al. [23] evaluated the effects of early armexercise training in patients recovering from acute respiratory failure requiring mechanical ventilation. Patients were randomised to either supported armexercise training plus general physiotherapy or to general physiotherapy alone. Patients undergoing supported armexercise training showed a greater improvement in exercise capacity, as assessed by an arm incremental test and an endurance test, compared with the control group. Posttraining maximal inspiratory pressure significantly improved in both groups. Incremental test isoworkload dyspnoea improved significantly in 
both groups, whereas isoworkload peripheral muscle fatigue, endurance test isotime dyspnoea and muscular fatigue improved significantly in the armexercise group but not in the control group. Incremental test and endurance test improvers were more frequently observed in the study group. Baseline maximal inspiratory pressure could discriminate between incremental and endurance tests improvers. Thus, the authors concluded that early upper-limb exercise training is feasible in patients recently weaned from mechanical ventilation and can enhance the effects of general physiotherapy. Baseline inspiratory muscle function is related to exercise capacity improvement.

\section{Outcome}

The BODE index [24], which integrates body mass index, airflow limitation (FEV1), dyspnoea and 6MWD, predicts mortality in COPD. Pulmonary rehabilitation improves some components of the BODE index. Cote et al. [25] hypothesised that changes in BODE may reflect the effects of PRP. To test this, a study was conducted on patients (BODE quartiles 2-4) who either declined, dropped out from or completed PRP.
BODE was determined at entry, after PRP, and at 1 and 2 years. Other outcomes included the length of stay for respiratory-related hospitalisations and mortality. At entry, the two groups had similar age and comorbidity but different BODE indices. After PRP, the BODE index improved by $19 \%$ and returned to baseline after 2 years. The BODE index worsened in the non-PRP group by $4 \%$ at 12 months and $18 \%$ at 2 years. Respiratory mortality at 2 years for PRP was $7 \%$, compared with $39 \%$ for the non-PRP group. Length of stay at 1 year for COPD decreased $20 \%$ in PRP, while it increased $25 \%$ in non-PRP. It was concluded that PRP improves BODE and is associated with better outcomes. The BODE index change after pulmonary rehabilitation provides valuable prognostic information.

\section{Conclusion}

In conclusion, the papers on pulmonary rehabilitation that were published in 2005 have provided useful information. The notion of biological effects of PRP, as reported in the paper by MERCKEN et al. [13], is extremely interesting, giving new research perspectives in the field.

\section{Educational questions}

1. Is respiratory muscle training always indicated in COPD patients?

2. Which of the following statements about the BODE index is true:

a) It is related to resource consumption.

b) It is related to survival.

c) It changes with pulmonary rehabilitation.

d) All of the above.

e) None of the comments above. 


\section{Suggested answers}

1. No

2. d

\section{References}

1. Pauwels RA, Buist AS, Calverley PMA, Jenkins CR, Hurd SS, on behalf of the GOLD Scientific Committee. Global strategy for the diagnosis, management and prevention of chronic obstructive lung disease. NHLBI/WHO global initiative for chronic obstructive lung disease (GOLD) workshop summary. Am J Respir Crit Care Med 2001; 163: 1256-1276.

2. Troosters $T$, Casaburi $R$, Gosselink $R$, Decramer M. Pulmonary rehabilitation in chronic obstructive pulmonary disease. Am J Respir Crit Care Med 2005; 172: 19-38.

3. Foglio K, Bianchi L, Bruletti G, Battista L, Pagani M, Ambrosino N. Long-term effectiveness of pulmonary rehabilitation in patients with chronic airway obstruction. Eur Respir J 1999; 13: 125-132.

4. Foglio K, Bianchi L, Ambrosino N. Is it really useful to repeat outpatient pulmonary rehabilitation programs in patients with chronic airway obstruction? A 2-year controlled study. Chest 2001; 119: 1696-1704.

5. Clini E, Foglio K, Bianchi L, Porta R, Vitacca M, Ambrosino N. In-hospital short-term training program for patients with chronic airway obstruction. Chest 2001; 120: 1500-1505.

6. Rossi G, Florini F, Romagnoli M, et al. Length and clinical effectiveness of pulmonary rehabilitation in outpatients with chronic airway obstruction. Chest 2005; 127: 105-109.

7. Verrill D, Barton C, Beasley W, Lippard WM. The effects of short-term and long-term pulmonary rehabilitation on functional capacity, perceived dyspnea, and quality of life. Chest 2005; 128: 673-683.

8. O'Donnell DE, Revill SM, Webb KA. Dynamic hyperinflation and exercise intolerance in chronic obstructive pulmonary disease. Am J Respir Crit Care Med 2001; 164: 770-777.

9. Diaz O, Villafranca C, Ghezzo H, et al. Role of inspiratory capacity on exercise tolerance in COPD patients with and without tidal expiratory flow limitation at rest. Eur Respir J 2000; 16: 269-275.

10. Plankeel JF, McMullen B, MacIntyre NR. Exercise outcomes after pulmonary rehabilitation depend on the initial mechanism of exercise limitation among non-oxygen-dependent COPD patients. Chest 2005; 127: 110-116.

11. Porszasz J, Emtner M, Goto S, Somfay A, Whipp BJ, Casaburi R. Exercise training decreases ventilatory requirements and exercise-induced hyperinflation at submaximal intensities in patients with COPD. Chest 2005; 128: 2025-2034.

12. Koechlin C, Couillard A, Simar D, et al. Does oxidative stress alter quadriceps endurance in chronic obstructive pulmonary disease? Am J Respir Crit Care Med 2004; 169: 1022-1027.

13. Mercken EM, Hageman GJ, Schols AMWJ, Akkermans MA, Bast A, Wouters EFM. Rehabilitation decreases exercise-induced oxidative stress in chronic obstructive pulmonary disease. Am J Respir Crit Care Med 2005; 172: 994-1001.

14. Clini E, Bianchi L, Foglio K, Porta R, Vitacca M, Ambrosino N. Effect of pulmonary rehabilitation on exhaled nitric oxide in patients with chronic obstructive pulmonary disease. Thorax 2001; 56: 519-523.

15. Casaburi $R$, Kukafka D, Cooper CB, Witek TD Jr, Kesten S. Improvement in exercise tolerance with the combination of tiotropium and pulmonary rehabilitation in patients with COPD. Chest 2005; 127: 809-817.

16. Bianchi R, Gigliotti F, Romagnoli I, et al. Chest wall kinematics and breathlessness during pursed-lip breathing in patients with COPD. Chest 2004; 125: 459-465.

17. Spahija J, de Marchie M, Grassino A. Effects of imposed pursed-lips breathing on respiratory mechanics and dyspnea at rest and during exercise in COPD. Chest 2005; 128: 640-650.

18. Lotters F, Van Tol B, Kwakkel $G$, Gosselink R. Effects of controlled inspiratory muscle training in patients with COPD: a metaanalysis. Eur Respir J 2002; 20: 570-576.

19. Mador MJ, Deniz O, Aggarwal A, Shaffer M, Kufel TJ, Spengler CM. Effect of respiratory muscle endurance training in patients with COPD undergoing pulmonary rehabilitation. Chest 2005; 128: 1216-1224.

20. Beckerman M, Magadle $R$, Weiner $M$, Weiner $P$. The effects of 1 year of specific inspiratory muscle training in patients with COPD. Chest 2005; 128: 3177-3182.

21. Martinez FJ, Vogel PD, Dupont DN, Stanopoulos I, Gray A, Beamis JF. Supported arm exercise vs unsupported arm exercise in the rehabilitation of patients with severe chronic airflow obstruction. Chest 1993; 103: 1397-1402.

22. Gigliotti F, Coli C, Bianchi $R$, et al. Arm exercise and hyperinflation in patients with COPD. Effect of arm training. Chest 2005; 128: $1225-1232$.

23. Porta $R$, Vitacca M, Gilè $L S$, et al. Supported arm training in patients recently weaned from mechanical ventilation. Chest 2005; 128: 2511-2520.

24. Celli BR, Cote CG, Marin JM, et al. The body-mass index, airflow obstruction, dyspnea, and exercise capacity index in chronic obstructive pulmonary disease. N Engl J Med 2004; 350: 1005-1012.

25. Cote CG, Celli BR. Pulmonary rehabilitation and the BODE index in COPD. Eur Respir J 2005; 20: 630-636. 